\title{
Optimization of Quality of Service Parameters for Dynamic Channel Allocation Scheme for Cellular Networks using Genetic Algorithim
}

\author{
Narendran Rajagopalan ${ }^{1}$, C.Mala ${ }^{2}$ \\ Computer Science and Engineering Department, National Institute of Technology, \\ Tiruchirapalli, Tamil Nadu, India - 620015 \\ ${ }^{1}$ narenrajl@gmail.com, ${ }^{2}$ cmalanitt@gmail.com
}

\begin{abstract}
As the spectrum for wireless transmission gets crowded due to the increase in the users and applications, the efficient use of the spectrum is a major challenge in today's world. A major affecting factor is the inefficient usage of the frequency bands. Interference in the neighboring cells affects the reuse of the frequency bands. In this paper, some of the quality of service parameters such as residual bandwidth, number of users, duration of calls, frequency of calls and priority are considered. This paper presents work based on the optimization of dynamic channel allocation using genetic algorithm (GA). This attempts to allocate the channel to users such that overall congestion in the network is minimized by reusing already allocated frequencies. The working of Genetic Algorithm which is used in the optimization procedure is also explained. The optimized channel is then compared with a non-optimized channel to check the efficiency of the genetic algorithm.
\end{abstract}

\section{Key words}

dynamic channel allocation, fixed channel allocation, cellular networks, genetic algorithm, crossover, fitness function

\section{Introduction}

The tremendous growth in mobile communication makes channel allocation critical due to the limited spectra available. The amount of spectrum left and the increasing cost have led to a limited spectrum available to each operator and hence making efficient use of it is required. The reuse of channel also becomes vital in this regard. Channels can be reused when the two stations do not interfere. Each operator can hence use all the spectra available to it by dynamically allocating frequencies to each cell in real time.

Studies have shown that under low traffic Dynamic Channel Allocation (DCA) fairs better in comparison to Fixed Channel Allocation (FCA) (also known as Static Channel Allocation (SCA)) [4][5][6]. However under high traffic conditions, FCA fairs better as there is always a constant 
International Journal of Next-Generation Networks (IJNGN) Vol.3, No.4, December 2011

stream of calls in high traffic, hence avoiding wastage of spectrum. Optimization isn't required in this case. But when there is medium to low traffic, Dynamic Channel Allocation can be used more efficiently than Fixed Channel Allocation [2].

Given the limitations and wastage of spectrum in FCA, it is obvious that the currently used fixed or static allocation scheme cannot accommodate the requirements of the devices in the near future. The solution to this may be found in DCA. Using DCA, the channel is allocated dynamically depending on the need of the service demands of the end user. This makes it more efficient than FCA.

But this leads to the issue of how the allocation is to be managed and optimized. In this paper, a Genetic Algorithm (GA) [1][2][8] is used to optimize the dynamicity of the channel. Taking three of the parameters for allocation and optimizing them using GA, an optimum usage scheme for these three parameters was obtained.

Genetic algorithms belong to the larger class of evolutionary algorithms (EA), which generate solutions to optimization problems using techniques inspired by natural evolution. It is a search heuristic that mimics the process of natural evolution such as inheritance, mutation, selection and crossover.

The rest of the paper is organized as follows. Section 2 presents fundamentals of Fixed Channel Allocation (FCA) and Dynamic Channel Allocation (DCA). In section 3, a description of Genetic Algorithm (GA) is given. The result of the optimization procedure is presented in section 4 . Conclusions and future work that can be derived from this are presented in section 5.

\section{Fundamental Channel Allocation Schemes}

Channel allocation schemes are basically used to allocate bandwidth and communication channels to base stations, access points and terminal equipments. The objective is to achieve maximum efficiency. Two types of strategies are followed -

- Fixed Channel Allocation (FCA)

- Dynamic Channel Allocation (DCA)

In FCA, each cell is given predetermined set of voice channel. A call can only connect by using an unused channel. If all the channels are occupied then the call is blocked in this system. This leads to congestion in traffic and some calls being lost when traffic gets heavy in some cells while some other cells may be idle at the same time.

A more efficient way of channel allocation would be Dynamic Channel Allocation (DCA) in which the voice channel is not allocated to cell permanently, but instead for every call request, the base station requests a channel from the Mobile Switching Center (MSC) [4][5]. The channel is then allocated following algorithms which accounts for the likelihood of future blocking within the cell. This requires the MSC to collect real time data on channel occupancy, traffic distribution, Radio Signal Strength Indications, etc. 
International Journal of Next-Generation Networks (IJNGN) Vol.3, No.4, December 2011

In this paper, three major factors which affect the channel allocation are taken namely Duration of Calls, Number of Users, Residual Bandwidth, Type of Service, Type of Users and Type of Calls for the purpose of optimization.

The fitness function with these three parameters is coined as -

$a+1 / b+c+1 / g+h+1 / i$

where,

Duration of Calls (a) : It is defined as the amount of time a user holds on to a certain call. This affects the allocation as knowledge of the amount of time the spectra is in use is vital for optimal allocation of the spectra to the users. The range of call times can be obtained from recorded history of usage by users over a period of time to find the average range of time that users hold the channel allocated to them.

Number of Users (b) : the number of users requesting allocation of frequency to make a certain voice or data call at a certain time. This is proportional to the number of users active in the given cell. This is also obtained using knowledge of the recent history of traffic in each cell owned by the operator.

Residual Bandwidth (c) : Residual bandwidth is the remaining spectra available to be allocated in each cell. The advantage of DCA over FCA takes effect as in FCA each cell is allocated with a given set of frequencies while in DCA the total spectrum can be allocated to any of the cells. The spectrum available to each cell is much higher in DCA in comparison to FCA. Residual Bandwidth or the number of free channels available in a cell is another Quality of Service parameter which can affect the service quality.

Type of Service (g) : depending upon whether it is a voice service or data service or multimedia service, this parameter can be used as a quality of service parameter to decide on channel allocation.

Type of Users (h) : Type of Users is a parameter differentiating regular users from roaming users. Roaming users must be given higher priority during channel allocation, so it is considered as one of the parameters for decision making.

Type of Calls (i) : Type of call is another parameter differentiating emergency calls from ordinary calls. It is one of the parameter which can affect the channel allocation decision.

\section{Genetic Algorithm}

Genetic Algorithms [1] are most commonly used as optimization algorithms. They are based on mechanics of natural selection (Darwin's Theory of Evolution). Natural Selection combines survival of the fittest and a randomized information exchange procedure to form a new generation from a previous generation in evolution. Genetic Algorithms follow the same procedures of using 
the fittest of the old generation as well as creation of new strings from previous generation through information exchange.

The mechanics of the genetic algorithm are simple involving only operations as copying of strings, swapping partial strings, etc. Initially, for applying genetic algorithm to a given problem, two things are needed namely - (i) an initial set of values which are basically strings of 0's and 1's (also known as individuals) based on the given parameters and (ii) a function known as fitness function, for evaluating how good or bad a particular individual is. This set of all the initial strings together is called as the initial population.

The initial population maybe generated in random or based on some initially assumed data for the given parameters. The string length of each individual depends on the problem at hand, the amount of optimization required and on the parameters as well. Some parameters may have many number of states while some others might not have that many. Hence, each parameter maybe allotted a certain number of bits based on the number of different states it can have. Its priority in the fitness function also matters. Priority is decided based on the effect of the parameter on the fitness function. If a small change in the state of a parameter affects the fitness of an individual drastically, then it is given a higher priority and vice versa. A parameter with higher priority maybe allotted more number of bits in order to increase the optimization of that parameter more which in turn increases the optimization of the whole procedure as such. The string length, as mentioned earlier, also depends on the amount of optimization required. If the string length is very high, i.e. a large number of states of the parameter are considered, and the population size is high, then all the variations of the states of the parameters maybe involved in the optimization procedure and hence might lead to a higher level of optimization. The population size is also decided based on the amount of optimization required for the given problem. More the population size, more is the variation in the generation, and hence more the optimization as more variations are analyzed during the optimization procedure. So as seen, the amount of optimization required decides almost all the factors involved in the initial population generation.

Once the initial population is generated, a simple set of operations is performed on it to generate successive populations which improve over time (hence, optimization). A simple genetic algorithm which does exactly that is composed of three operations - (i) Reproduction, (ii) Crossover and (iii) Mutation.

Reproduction [1][8] is the process in which individuals are copied according to their fitness, which is calculated using the fitness function. Individuals with a higher fitness function (in a maximization problem) are copied over to the next generation while those with the least fitness function are neglected or discarded. Copying an individual according to its fitness leads to a higher probability of it contributing a better offspring (having a higher fitness) in the next generation. Copying of individuals is achieved based on its percentage contribution to the sum of all the fitness functions put together.

Crossover [1][8] is the process of information exchange. After reproduction is over, the set of individuals are then paired in random and mated. Mating is the process where each pair undergoes swapping of all the characters from one string to its mate from a randomly decided position in the string. This random point is known as crossover point. This is the primary step involved in the evolutionary process of genetic algorithm. Crossover combined with reproduction leads to effective optimization through recombination of individuals. 
International Journal of Next-Generation Networks (IJNGN) Vol.3, No.4, December 2011

Mutation [1][8] is the process of altering of value of an individual in random, i.e., changing the value of a random character in an individual from 0 to 1 or vice versa. It is implemented to ensure that there is no loss of optimization if by chance, all of the population contains 0's or 1's at a particular location which will just be carried forward to the next generation as such if only reproduction is used and crossover leading to incomplete optimization. The frequency of mutation is usually of the order of one mutation per thousand reproduction cycles, which is similar to the mutations rates in natural selection.

A simple example [1] to demonstrate all the functions of a genetic algorithm is given below. Assume the initial population as shown and the let the fitness function be $f(x)=x^{2}$

Table. 1. Example to demonstrate Genetic Algorithm [1]

\begin{tabular}{|c|c|c|c|c|c|}
\hline $\begin{array}{c}\text { Individual } \\
\text { No. }\end{array}$ & $\begin{array}{c}\mathbf{x} \\
\text { Values }\end{array}$ & $\begin{array}{c}\text { Population } \\
\text { (binary } \\
\text { representation of } \\
\mathbf{x} \text { ) }\end{array}$ & $\begin{array}{c}f(x)= \\
x^{2}\end{array}$ & $\begin{array}{l}\text { Percentage } \\
\text { Contribution } \\
\text { to Sum(p) }\end{array}$ & $\begin{array}{c}\text { Copies } \\
\left(p^{\star} 4\right)\end{array}$ \\
\hline 1. & 13 & 01101 & 169 & $14 \%$ & $0.56 \approx 1$ \\
\hline 2. & 24 & 11000 & 576 & $49 \%$ & $1.96 \approx 2$ \\
\hline 3. & 8 & 01000 & 64 & $6 \%$ & $0.24 \approx 0$ \\
\hline 4. & 19 & 10011 & 361 & $31 \%$ & $1.24 \approx 1$ \\
\hline
\end{tabular}

The initial population contains a population of 4 individuals. Their fitness is calculated using the fitness function as shown. After the fitness is calculated for each individual, the sum of all the fitness functions of the individuals is found (here it is 1170). Then, the percentage contribution for each individual to the sum is found.This percentage contribution multiplied by the total population size, gives the number of copies of each of the individuals present in the following generation.The result of this operation on the above set of individuals leads to 2 copies of individual 2 and individual 3 being discarded giving rise to the intermediate population of :

$$
\begin{array}{lllll}
0 & 1 & 1 & 0 & 1 \\
1 & 1 & 0 & 0 & 0 \\
1 & 1 & 0 & 0 & 0 \\
1 & 0 & 0 & 1 & 1
\end{array}
$$

At this point, the procedure of crossover is implemented on this intermediate population set. Suppose, individual 1 is paired with individual 2 and individual 3 with individual 4 . Assume the crossover points are 4 and 1 respectively. Then, the crossover occurs as:

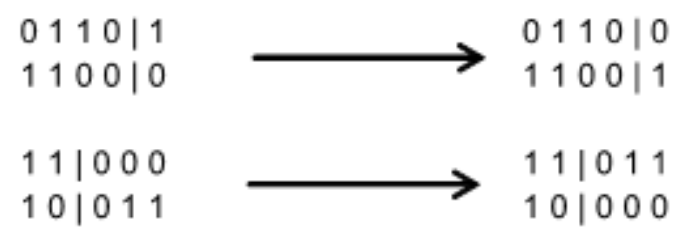


International Journal of Next-Generation Networks (IJNGN) Vol.3, No.4, December 2011

Mutation then occurs at a probability of 0.0001 which is highly improbable for one reproduction cycle. But if mutation were to occur, at say individual 1, then it would be something like:

\section{$01101 \longrightarrow 11101$}

At this point, a new population set is generated. The procedure of Reproduction, Crossover and Mutation is carried out repeatedly till a termination condition for the optimization is reached. The termination condition maybe a highest level of fitness reached or fixed number generations reached or satisfaction of certain constraints which is decided depending on the problem. Once the termination condition is reached, the population that is generated after the last iteration is said to be optimized.

In our tests, the following procedures were performed as a part of the genetic algorithm that was implemented -

- Initial population is randomly generated.

- Each individual is evaluated as per the fitness function.

- Repeat on each generation until termination -

(a) Best individuals are selected for reproduction.

(b) New individuals are obtained through crossover and mutation.

(c) New individuals are evaluated as per the fitness function.

(d) Least-fit individuals of the population are replaced with the new individuals.

\subsection{Proposed optimization scheme for Dynamic Channel Allocation using Genetic Algorithm}

In the proposed scheme, an initial random population of 100 is generated with a random number generator. Each of the three parameters were given equal priority and taken as 8-bit values. Hence, 100 random numbers of 24-bit string length was generated to be the initial population.

The 8-bit representation of each of the parameters is as follows -

- A periodicity value of 15 seconds is represented by the 00000001,30 seconds as 00000010 and so on.

- 1 user requesting a channel is represented as 00000001,2 users as 00000010 and so on.

- Residual bandwidth is represented by percentage of bandwidth remaining, i.e. $100 \%$ bandwidth remaining is represented by 11111111 and $0 \%$ bandwidth as 00000000 and so on. 
International Journal of Next-Generation Networks (IJNGN) Vol.3, No.4, December 2011

Table. 2. Initially generated random population

\begin{tabular}{|l|l|l|l|l|l|l|l|}
\hline $\begin{array}{l}\text { S1. } \\
\text { No }\end{array}$ & $\begin{array}{l}\text { Duration } \\
\text { of Call(a) }\end{array}$ & $\begin{array}{l}\text { Number of } \\
\text { Users(b) }\end{array}$ & $\begin{array}{l}\text { Residual } \\
\text { Bandwid } \\
\text { th(c) }\end{array}$ & Priority(d) & $\begin{array}{l}\text { Time of } \\
\text { call(e) }\end{array}$ & $\begin{array}{l}\text { Frequency } \\
\text { of Call(f) }\end{array}$ & F(x) \\
\hline 1 & 52 & 105 & 67 & 245 & 195 & 60 & 314.03 \\
\hline 2 & 8 & 87 & 253 & 237 & 48 & 25 & 309.06 \\
\hline 3 & 213 & 242 & 162 & 249 & 55 & 37 & 430.04 \\
\hline 4 & 117 & 234 & 53 & 164 & 117 & 200 & 287.02 \\
\hline 5 & 115 & 109 & 146 & 196 & 232 & 139 & 493.02 \\
\hline 6 & 210 & 108 & 88 & 192 & 250 & 39 & 548.04 \\
\hline
\end{tabular}

\begin{tabular}{|l|l|l|l|l|l|l|l|}
\hline 7 & 95 & 110 & 184 & 82 & 77 & 245 & 356.03 \\
\hline 8 & 202 & 57 & 125 & 154 & 70 & 52 & 397.04 \\
\hline 9 & 40 & 188 & 153 & 224 & 23 & 76 & 216.03 \\
\hline 10 & 111 & 185 & 159 & 118 & 25 & 172 & 295.02 \\
\hline 11 & 128 & 81 & 147 & 97 & 177 & 209 & 452.03 \\
\hline 12 & 34 & 204 & 202 & 32 & 121 & 102 & 357.05 \\
\hline 13 & 101 & 150 & 109 & 130 & 164 & 42 & 374.04 \\
\hline 14 & 69 & 221 & 165 & 157 & 9 & 253 & 243.01 \\
\hline 15 & 177 & 206 & 150 & 38 & 127 & 252 & 454.04 \\
\hline 16 & 180 & 214 & 28 & 218 & 87 & 193 & 295.01 \\
\hline 17 & 62 & 34 & 63 & 212 & 65 & 126 & 190.04 \\
\hline 18 & 196 & 101 & 47 & 61 & 134 & 180 & 377.03 \\
\hline 19 & 206 & 166 & 243 & 67 & 158 & 118 & 607.03 \\
\hline 20 & 161 & 218 & 119 & 174 & 219 & 90 & 499.02 \\
\hline
\end{tabular}

Table. 2. gives a sample of the initial population that is generated which is then used for optimization.

The $f(x)$ values were then calculated for each of the individuals of the initial population. The sum of the $f(x)$ values was calculated and the percentage contribution of each of the individuals was calculated. The individuals with a higher contribution percentage as compared to the rest were favored and the ones with a lower percentage contribution were given lesser preference during the selection procedure for mating for the next generation. The selected individuals were paired in random with another individual and mating was carried out. Crossover with a probability of 1 and mutation with a probability of 0.001 were the operations carried out during the mating procedure. This is the point in the algorithm where optimization, or evolution as is the case here, occurs. The 
International Journal of Next-Generation Networks (IJNGN) Vol.3, No.4, December 2011

best of the individuals were taken and then mated to get individuals with the favorable qualities of both the parent individuals. This leads to a new generation of individuals with a higher average $\mathrm{f}(\mathrm{x})$ value.

This generational process is repeated until a termination condition has been reached. In our tests, a fixed number of generations was used and the optimization procedure was terminated after 10 iterations. Table. 3. gives the result of the optimization of the sample initial population taken in Table. 2.

In order to apply genetic algorithm to the problem, The parameters have to be encoded to strings. The parameters are represented as eight bit strings. Some of the parameter values are obtained from the history of the caller. The parameter 'a' is the time duration, a user has called. Every 10 seconds is assumed to be one unit. Hence a value of $\mathrm{a}=25$, would imply the duration of the call to be 250 seconds. The parameter 'Type of Service' is higher if the value of $g$ is higher. Hence if $\mathrm{g}=100$, then it has a higher priority than $\mathrm{g} 2=65$, but lesser than $\mathrm{g} 3=150$. Depending upon the density of users in a cell, a unit is selected like 10 users as one unit. Hence a value of $b=45$ would indicate 450 users. A fitness function is needed and initial population as seed is required. The fitness function is a maximization function. The initial values for the strings are randomly generated and tabulated as shown in table below. Each parameter is represented as string of eight bits each.

\section{Performance Analysis}

Matlab is used for conducting performance analysis. For each case study, any two parameters are constantly increased at a predetermined interval and the performance of Heuristic method and Genetic Algorithm is recorded varying other parameters. Throughput in the figures is the percentage of best theoretical case to the throughput achieved by Genetic Algorithm.

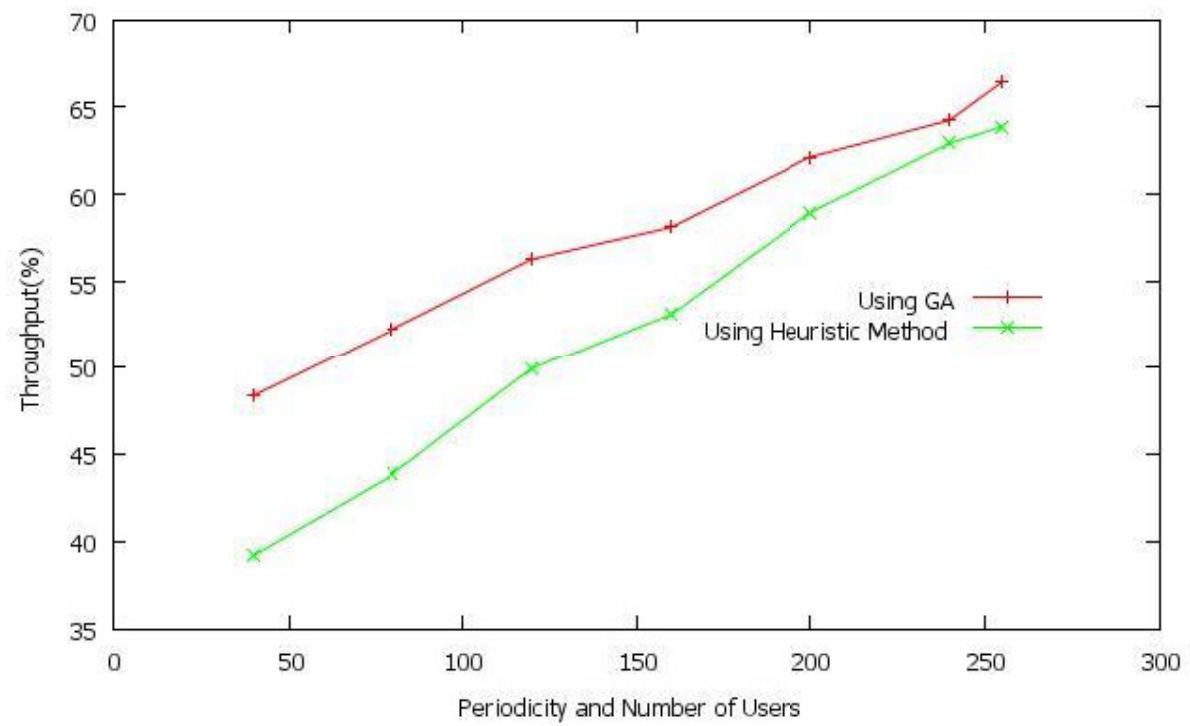

Figure 1. Performance of Heuristic Method and Genetic Algorithm with Parameters Periodicity and Number of Users fixed at different Intervals 
International Journal of Next-Generation Networks (IJNGN) Vol.3, No.4, December 2011

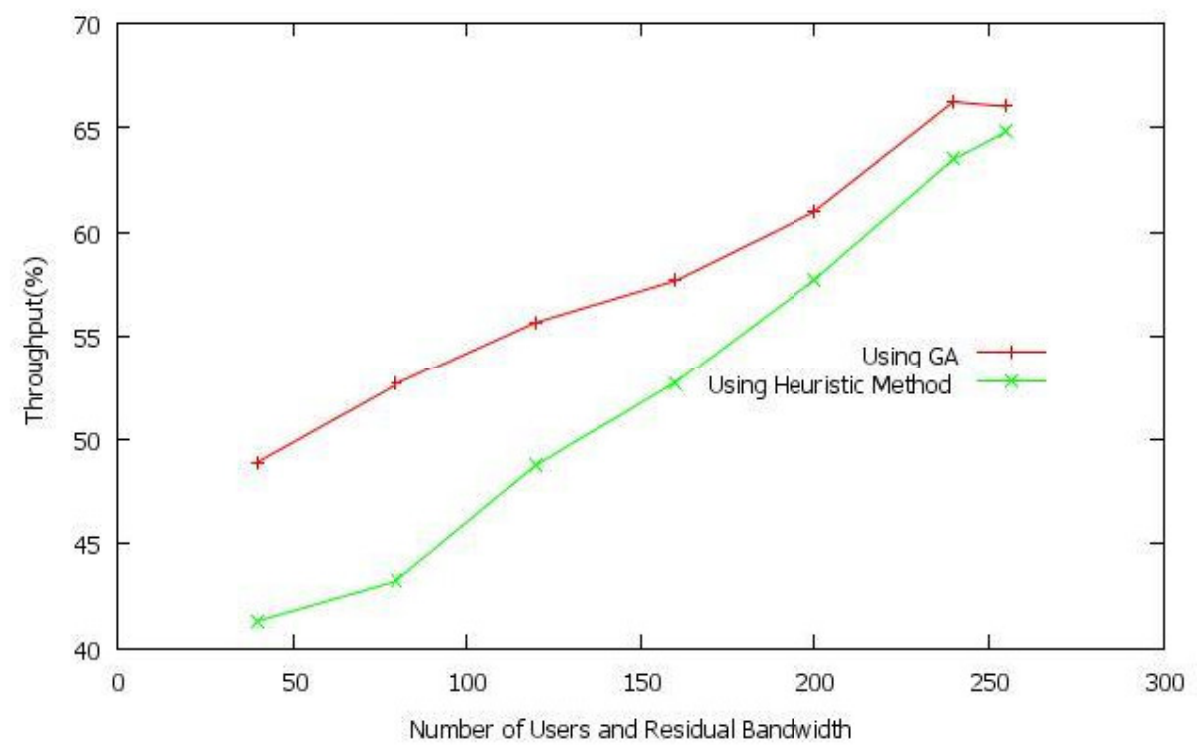

Figure 2. Performance of Heuristic Method and Genetic Algorithm with Parameters Number of Users and Residual Bandwidth fixed at different Intervals

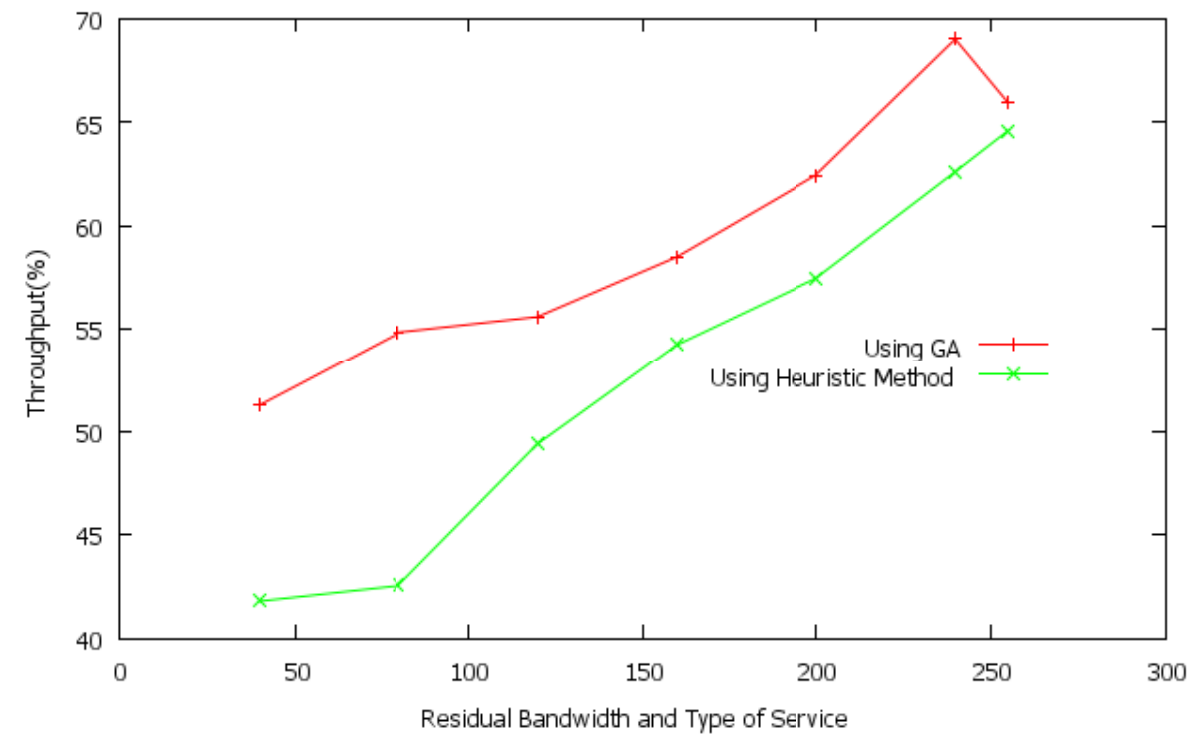

Figure 3. Performance of Heuristic Method and Genetic Algorithm with Parameters Residual Bandwidth and Priority fixed at different Intervals 
International Journal of Next-Generation Networks (IJNGN) Vol.3, No.4, December 2011

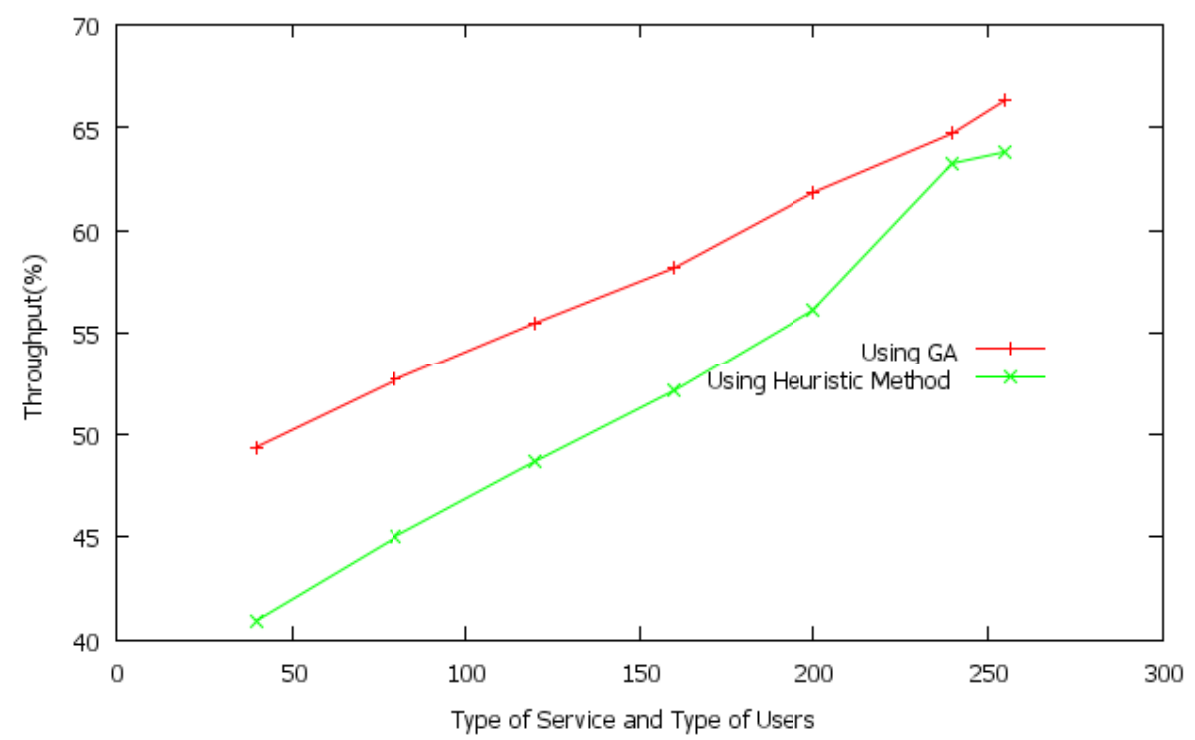

Figure 4. Performance of Heuristic Method and Genetic Algorithm with Parameters Priority and Time of Call fixed at different Intervals

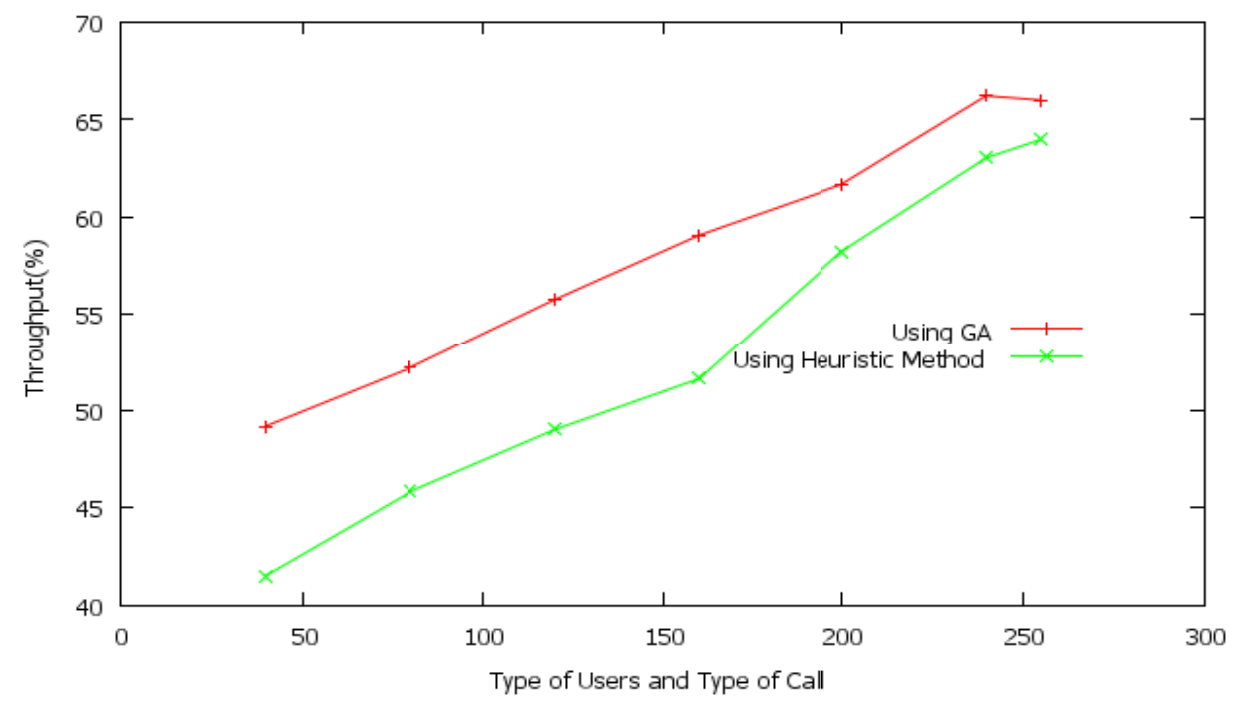

Figure 5. Performance of Heuristic Method, Genetic Algorithm with Parameters Time of Call and Frequency of Calls fixed at different Intervals

\section{Conclusion}

The channel allocation problem is analyzed with quality of service parameters like Call Duration, Number of Users, Residual Bandwidth, Priority, Time of Call and frequency of calls. In this paper, heuristic method, genetic algorithm and artificial neural network are applied to these parameters. The graphs in figure 1 to figure 5 shows that throughput of Genetic algorithm is better than heuristic method. 
International Journal of Next-Generation Networks (IJNGN) Vol.3, No.4, December 2011

\section{References}

[1] Goldberg, D.E.: Genetic Algorithms in Search, Optimization, and Machine Learning. Pearson Education, India (2004)

[2] Tsai, C., Lin, F., Tsai, C.: An efficient GA-based approach for fixed channel assignment in cellular radio networks, Computational Intelligence in Robotics and Automation, 2003. Proceedings. 2003 IEEE International Symposium on Vol. 1, Issue 16-20 pp. 384-389, July 2003.

[3] Martinez, D., Andrade, A.G., Martinez, A.: Interference-Aware Dynamic Channel allocation scheme for cellular networks, IEEE Press

[4] Qing, Z., Sadler, M.: A Survey of Dynamic Spectrum Access, IEEE Signal Processing Magazine, Vol.70, No.5, pp. 79-89 May 2007.

[5] Granelli, F., Przemyslaw, P., Venkatesha, P., Hoffmeyer, J.: Standardization and Research in Cognitive and Dynamic Spectrum Acess Networks: IEEE SCC41 efforts and Other Activities, IEEE Communications Magazine, vol.48, No.1, pp. 71-79, January 2010.

[6] Katzela, I., Naghshineh. M.: Channel assignment schemes for cellular mobile telecommunication systems: a comprehensive survey. IEEE Personal Communications, 3(3):10-31, Jun 1996.

[7] Kaabi, F., Ghannay, S., and Filali, F.: Channel allocation and routing in Wireless Mesh Networks:A Survey and qualitative comparison between schemes, International Journal of Wireless and Mobile Network, Vol.2, No.1, pp. 132-151, February 2010.

[8] Wikipedia, http://www.wikipedia.org/ 